\title{
Ethiopia's Health Extension Program: Improving Health through Community Involvement
}

\author{
Hailom Banteyerga PhD
}

\begin{abstract}
The Health Extension Program is one of the most innovative community-based health programs in Ethiopia. It is based on the assumption that access to and quality of primary health care in rural communities can be improved through transfer of health knowledge and skills to households. Since it became operational in 2004-2005, the Program has had a tangible effect on the thinking and practices of rural people regarding disease prevention, family health, hygiene and environmental sanitation. It has enabled Ethiopia to increase primary health care coverage from $76.9 \%$ in 2005 to $90 \%$ in 2010.
\end{abstract}

KEYWORDS Family health, health education, health program, disease prevention, health policy, health priorities, environmental hygiene, sanitation, Millennium Development Goals, Ethiopia

\section{INTRODUCTION}

Ethiopia is one of the poorest countries in the world with a US\$330 per capita Gross National Income in 2009. The health system remained weak until the country introduced a 20 -year health sector development program in 1977, which is being implemented in four phases.[1] The civil war waged during the 17 years of military rule from 1974 to 1991 destroyed the health delivery system, and the country faced serious economic problems leading to shortages of skilled health human resources, pharmaceuticals, and health service delivery facilities.

These conditions, coupled with harmful traditional practices such as female genital mutilation, meant that Ethiopia continued to suffer from a high disease burden, of which $60 \%$ was preventable.[2] Maternal and childhood mortality are still among the highest in Africa: under-five mortality was 210 per 1000 children in 1990, falling to 104 per 1000 in 2009.[3] Maternal mortality was 673 per 100,000 live births in 2005 , dropping to 470 per 100,000 in 2008.[4] Most of the causes of child and maternal mortality are preventable. Lack of information and knowledge on prevention, shortage of health facilities within accessible distance, and insufficient skilled health personnel to deliver services have been contributing factors to high rates. Malaria, HIVI AIDS, TB, waterborne diseases, and respiratory infections remain among the top ten killer diseases.[2]

The Federal Ministry of Health (FMOH) of Ethiopia launched the Health Extension Program (HEP) in 2003 and it became operational with the 2004-2005 graduation of 7136 Health Extension Workers (HEW), trained to work mainly in disease prevention and health promotion in rural villages.[5] The program was expected to help accelerate the country's progress in meeting Millennium Development Goals (MDG) 4, 5 and 6 (reduce child mortality; improve maternal health; combat HIVIAIDS, malaria and other diseases).[6] Now it is the country's major health program: by 2010 , there were $30,578 \mathrm{HEW}$ serving almost all villages in rural areas where sedentary farming rather than nomadism is the norm.[2]
This paper is based primarily on three major studies conducted at Miz-Hasab Research Center (MHRC): The Last Ten KilometersL10K (2008);[7] System Wide Effect of the Fund (SWEF, 2010),[8] and Good Health at Low Cost (GHLC).[9] These found that HEP accelerated access to primary health care and had an impact in reducing communicable diseases and maternal and childhood mortality. We share this experience for the benefit of other countries in the same economic stratum.

What is the Health Extension Program (HEP)? HEP is a community-based health service delivery program whose educational approach is based on the diffusion model, which holds that community behavior is changed step by step: training early adopters first, then moving to the next group that is ready to change. Those resistant to change would gradually be conditioned to change because of changes in their environment.[10] HEP assumes that health behavior can be enhanced in communities by creating model families that others will admire and emulate.[5,10]

HEP has four health subprograms: disease prevention, family health, environmental hygiene and sanitation, and health education and communication;[5] these correspond to the elements of primary health care coverage as defined in the Alma Ata Declaration.[11] Every village with 5000 residents builds a health post. Two female HEWs who have completed tenth grade are recruited from the same community and trained in HEP modules for one year, after which they return home as salaried frontline health care staff. According to the $\mathrm{FMOH}$ :

HEWs are the first point of contact of the community with the health system, delivering integrated preventive, promotive and curative health services, with a special focus on maternal and child health. The aim is to ensure continuity of care throughout the lifecycle (adolescence, pregnancy, childbirth, postnatal period, and adulthood) and also between places of care giving services, and clinical-care settings-having wide implications for the achievement of MDGs in Ethiopia.[12]

HEWs' major task is increasing knowledge and skills of communities and households to deal with preventable diseases and be able to access services available at clinics and hospitals. Since HEP considers maternal and child health tracer indicators of good health, HEWs give special attention to family health. In addition to conducting preventive family health education and sanitation, they can supervise intake of community Directly Observable Treatment-Short Course (DOTS) for TB and antiretroviral treatment for HIVIAIDS; conduct rapid diagnostic tests for malaria and administer malaria drugs; attend uncomplicated childbirth; refer patients to nearby health centers; and collect vital statistics. HEWs are not allowed to administer antibiotics.[5]

One of an HEW's first tasks is to recruit Voluntary Community Health Workers (vCHWs) to help implement HEP. These are individuals who have had experience in community-based health services, such as those trained as community-based health workers prior to HEP. 
The first families to be trained are selected by the local government (kebele) administration, HEWs, vCHWs and community leaders based on criteria related to their earlier participation in community health activities and readiness to enroll in the training. Women in the selected families take the lead role in the training, recognizing that women take more responsibility in family care. Other families are selected for training when the first batch graduates. Each group receives 96 hours of training, involving face-toface teaching and household visits in four modules corresponding to the four HEP subprograms: prevention of communicable diseases, family health, environmental and household sanitation, and health education. Formal training continues until all households graduate. HEWs and vCHWs follow up with households regularly.

\section{Key Resources for Assessing HEP Impact}

The Rapid Appraisal of Health Extension study was sponsored by the L10K Project of John Snow International (JSI). The study is based on qualitative data collected from four major regions: Oromia, Tigray, Amhara and Southern Nations, Nationalities and Peoples Region.[7]

The System Wide Effect of the Fund (SWEF) is a study sponsored by United States Agency for International Development (USAID) to assess the effect of global health initiatives, particularly of the Global Fund to Fight AIDS, Tuberculosis and Malaria on national health systems and policy. Three rounds of SWEF studies have been conducted between 2004 and 2010 by Abt Associates and MHRC.[8]

The Good Health at Low Cost study is a five-country study coordinated by the London School of Hygiene and Tropical Medicine and sponsored by the Rockefeller Foundation. It selected five low-income countries, including Ethiopia, and aimed to see how they have achieved good outcomes in health.[9]

The diffusion model assumes that change happens gradually. As model families change their health practices, they influence their neighbors and friends formally in venues such as community meetings, and informally when they get together for social activities such as coffee ceremonies and in idir and mahber, community associations formed for mutual practical support (the former for funeral services, the latter for activities such as harvesting and home-building). The social context gets modified by facilitating discussion of the content of the four HEP modules. As HEP is implemented, household arrangement of rooms and utensils and the availability of pit latrines change the physical structure of the household. One vCHW supervises 10 to 20 households and $70 \%$ of HEWs' time is allocated to household visits.

HEP is an expression of the government's political will regarding health. All government sectors, local leaders, and communities are required to collaborate in implementation of HEP programs: health post construction; selection of vCHWs and model families; mobilization of communities during immuniza- tion campaigns; and construction of sanitary facilities. Schools, agriculture extension agents, community associations, as well as other social structures are involved in disseminating HEP messages and promoting good health practices. Development partners are encouraged to strengthen HEP implementation activities, by providing health materials and supplies, training, and monitoring.

HEP assumes that communities are owners, producers and multipliers of health. The roles of the health provider and other stakeholders are to enable households and communities to lead a healthy life by (i) building their capacities and skills in disease prevention and health management; (ii) providing basic health tools and commodities such as family planning methods, vaccines, oral rehydration salts, community DOTS, and malaria drugs and nets; (iii) attending childbirth; and (iv) providing timely referral to specialized care for those who need it.

\section{WHAT HAS HEP DONE SO FAR?}

Attribution of results to specific programs is always difficult because of the multiplicity of factors and actors in the social environment, but tangible improvements in key health indicators have been observed since HEP's implementation began,[2,13] supporting our conclusion that HEP is an effective approach to promoting good health in rural communities. It is now present in all rural agrarian areas and is being expanded to include pastoralist and urban areas.

Credit for these improvements (Table 1) must be shared with global health initiatives that are major players in the implementation of the health sector development program. The Global Fund to Fight HIVIAIDS, Tuberculosis and Malaria; the President's Emergency Plan for AIDS Relief; and the World Bank have been major players in health for the last ten years. This has helped the work of the FMOH in HEP implementation.

Table 1: Progress in key health indicators in Ethiopia, 2005-2010

\begin{tabular}{l|r|r|}
\multirow{2}{*}{ Primary health care coverage } & \multicolumn{2}{|c|}{ Year } \\
\cline { 2 - 3 } & $\mathbf{2 0 0 5}(\mathbf{\%})$ & $\mathbf{2 0 1 0}(\mathbf{\%})$ \\
\hline Expanded Program of Immunization (EPI) & 76.9 & 90.0 \\
\hline Contraceptive acceptance rate & 76.8 & 81.6 \\
\hline Antenatal coverage & 37.9 & 56.2 \\
\hline HIV prevalence & 50.4 & 67.7 \\
\hline
\end{tabular}

Source: Federal Ministry of Health, Ethiopia

Analyzing qualitative data collected from the L10K study, HEP's main achievements (as expressed by those benefited) can be summarized as follows:[7]

HEP has created greater awareness of how to prevent communicable diseases such as malaria, tuberculosis, HIVIAIDS and waterborne disease. Community members interviewed during the L10K study reported that malaria is no longer epidemic. They say that diarrhea and other waterborne diseases and eye infections are decreasing:

Our children are no longer suffering from diarrhea, eye infections and malnutrition. We keep them clean and feed them as per the advice given to us by the HEWs. (L10K: focus group discussion, Tigray) 
Community and household attitudes toward HIVIAIDS and those living with HIVIAIDS are changing, as have practices related to prevention of infectious diseases in general. For example, the L10K appraisal reported that communities and households openly discussed HIVIAIDS and cared for those living with the virus. Knowledge and behavioral practices toward prevention of sexually transmitted infections have improved. Informants said that that their lifestyles have changed because of HEP:

We learnt from HEWs that by doing simple things at home we can protect ourselves from diseases. Keeping our houses, children and environment clean protects us from deadly diseases. These are simple things that anybody can do. Since we started keeping our homes clean, our children and family members have become healthy. (L10K: focus group discussion participant, Southern Region)

Beneficiaries reported changed attitudes and behavioral practices in preventive aspects of maternal and child health. Community informants and district and regional program coordinators say that mothers and children are regularly monitored by HEWs. Access to family planning, antenatal and postnatal care services has improved,[2] and maternal and childhood disease incidences have decreased as a result.

Disease incidence is minimized enormously, childhood and maternal deaths are reduced and the community has started to use family planning programs efficiently; the number of women using contraceptives and accessing pre- and postnatal care services is increasing. (L10K: HEW, Amhara)

Community informants in the L10K study all agreed that infant feeding habits such as breast feeding and use of supplements have improved; the number of children and mothers immunized has increased, and practices in reporting sick children to HEWs have shown improvement. The study team observed such trends in the charts of the visited health posts. A community informant says:

I really have benefited from HEP. I used to give birth every year. Now I breast feed, take contraceptives and avoid getting pregnant [so often]. (L10K: community informant, Oromia)

$\mathrm{FMOH}$ statistics on health and health-related indicators show that antenatal coverage increased from $67.7 \%$ in $2008-2009$ to $71.4 \%$ in 2009-2010 and postnatal coverage from $34.3 \%$ to $36.2 \%$ in the same period.[14]

HEP has improved sanitation and increased access to safe and clean drinking water from $35.9 \%$ in $2004-2005$ to $66.2 \%$ in 2009-2010 nationally,[2] when access to safe excreta disposal reached $60 \%$. The research team of the L10K appraisal observed that households visited had pit latrines; separated animal sheds; improved kitchens, bedrooms and living rooms; and cleanly managed drinking water and household goods. A community informant says:

We have started drinking clean and safe water, keeping our houses and environment clean, using pit latrines, separating animal sheds from our homes, [and] using a separate kitchen. (L10K: community informant, Tigray)
The diffusion model as an approach to health behavioral change is working. HEP's impact is visible in the way people live in their communities and from what local stakeholders say:

They [HEWs] are influencing the community...teaching the community. We used to suffer from different diseases. Now we are learning to take preventive measures and we are not experiencing any epidemics. (L10K: community leader, Oromia)

HEP has created synergy among public sector, community, and development partners. Local leaders, religious leaders, and associations of youth, women and farmers actively participate during construction of pit latrines, vaccination, and community meetings. Development partners are assisting in the training of HEWs and by supplying health posts with essential drugs and supplies. A development partner informant interviewed for the L1OK appraisal says:

HEP has created good ground for collaboration with partners. During the implementation of health activities at the kebele [the smallest unit of government structure] level, development partners participate in HEP in terms of finance, supply of commodities and materials, construction of health posts, and supplies of drugs and vaccines.

The government is encouraging partners in health to work at the village level with the motto: All roads lead to the Health Extension Program!

\section{CHALENGES}

Nevertheless, HEP has experienced some challenges. Graduation of model families did not happen as planned. It was expected that within three years of implementation, all households would have graduated;[15] however, due to travel time between households and competing demands for family members' time for farming activities, model family training is taking longer than anticipated.

Also, use of voluntary community health workers appears hard to sustain without some material compensation for extra services rendered to communities. With the government's policy of alignment of health activities, HEP is becoming the focal point of health programming, so increasingly, development partners are also executing their programs through HEP, creating heavier workloads for HEWs. Although the increased integration is positive, HEW burnout has been observed as a result.

Career advancement is also a critical issue for HEWs and efforts are being made to address it.

\section{CONCLUSIONS}

Ethiopia's HEP has shown tangible positive impacts on community health, in disease prevention, family health, and environmental hygiene and sanitation. The government has made HEP the foundation of the country's emerging new health system. Local government and community participation is gaining momentum, and the roles and interests of development partners are crystallizing. In order to strengthen HEP, FMOH has embarked on training health officers and midwives. Cold storage for vaccines is also being addressed through making refrigerators available. 
HEP demonstrates that instead of sticking to traditional health provider and medication-oriented models, context-sensitive and affordable functional models and approaches could be developed to expand primary health care services. Development partners in health were skeptical of HEP at first, but it has now proved to be a more effective approach to preventing disease and instilling skills and knowledge than the village clinic-based approach, which focused more on curative services with less attention to prevention. HEP is now at the center of global health initiatives directed to villages and districts in Ethiopia. Ethiopian health officials expect to meet MDGs 4 and 6 and to have made progress toward MDG 5 by 2015 .

What is more important is that HEP has shown that population behavior patterns can be changed to be more favorable to good health. With strong political will and a sense of purpose, lowincome countries can use innovative approaches to achieve universal coverage of primary health care. -1 -

\section{REFERENCES}

1. Bachrach P. Revision and Finalization of the Program Action Plan of Ethiopia's Health Sector Development Program [Internet]. Addis Ababa: Federal Ministry of Health (ET); 1998 Jul [cited 2011 May 24]. 17 p. Available from: http://pdf. usaid.gov/pdf_docs/PDABR020.pdf

2. Federal Ministry of Health (ET), Planning and Programming Department. Health and Health related indicators [Internet]. Addis Ababa: Federal Ministry of Health (ET). 2004 Dec [cited 2011 May 24]. 59 p. Available from: http://cnhde. ei.columbia.edu/indicators/Health_Indicators96. pdf

3. UNICEF. Ethiopia Statistics. Basic Indicators 2009 [Internet]. New York: UNICEF; 2010 [updated 2010 Mar 2; cited 2011 May 24]. Available from: http://www.unicef.org/infobycountry/ethiopia statistics.html

4. A bdela A. Maternal mortality trend in Ethiopia. Ethiop J Health Dev [Internet]. 2010 [cited 2011 May 24];24(Special Issue 1):115-22. Available from: http://ejhd.uib.no/ejhd-v24-sn1/115\%20 Maternal\%20Mortality\%20Trend\%20in\%20 Ethiopia.pdf

5. Federal Ministry of Health (ET). Health Extension Program in Ethiopia: Profile [Internet]. Addis Ababa: Health extension and Education Center; 2007 [cited 2011 May 24]. 27 p. Available from: http://www.ethiopia.gov.et/English/MOH/ Resources/Documents/HEW\%20profile\%20 Final\%2008\%2007.pdf

6. United Nations. The Millennium Development Goals Report 2008 [Internet]. New York: United Nations Department of Economic and Social
Affairs; 2008 [cited 2011 Apr 19]. 52 p. Available from: http://www.undp.org.af/publications/Key Documents/MDG_Report_2008_En.pdf

7. Banteyerga H, Kidanu A. Rapid Appraisal of Health Extension program: Ethiopia Country Report. Addis Ababa: The L10K project and MHRC; 2008.

8. Banteyerga $\mathrm{H}$, Kidanu A, Hochkiss D, De $\mathrm{S}$, Tharaney M, Callaham K. The System Wide Effects of the Scale Up of HIVIAIDs, Tuberculosis, and Malaria Services in Ethiopia [Internet]. Bethesda: Health systems 20/20 Project; 2010 Oct [cited 2011 May 24]. 89 p. Available from: www.ghinet.org/downloads/Ethiopia_SWEF_ ReportOct_2010_FIN.pdf

9. Banteyerga $H$, Kidanu A, Conteh L, McKee M. Ethiopia Chapter on Good health at Low Cost. A study conducted by LSHTM and Miz-Hasab Research Center, along with other four countries, commissioned by the Rockefeller Foundation. Forthcoming; 2011.

10. Ulin PR, Robinson ET, Tolly EE. Qualitative Methods in Health: A field Guide for Applied research. San Francisco: Jossey Bass; 2005.

11. World Health Organization. Declaration of Alma-Ata: International Conference on Primary Health Care, Alma-Ata, USSR, Sept 6-12, 1978 [Internet]. Geneva: World Health Organization; 1978 [cited 2011 May 24]. 3 p. Available from: http://www.who.int/hpr/NPH/docs/declaration_ almaata.pdf

12. Roman T, Accorsi S, Akalu T, Vella V, Mamo D. Assessing the performance of the health sector: achievements and challenges in EFY 2001
[G.C.2008/9]. Policy and Practice: information for action. Quarterly Health Bulletin. Addis Ababa: Federal Ministry of Health (ET); 2010.

13. Health Sector Development Program III: Annual Performance Report [Internet]. Addis Ababa: Federal Ministry of Health (ET); 2011 [cited 2011 May 24]. Available from: www.moh.gov.et/index. php?option=com_remository\&Itemid $=59 \&$ func $=$ fileinfo\&id=11

14. Federal Ministry of Health (ET). Office of Public Relations. The Health Service Before and After 1983 Ec (1991 GC): A summary of achievements from 1991 to 2010. Addis Ababa: Federal Ministry of Health (ET); 2010.

15. Federal Ministry of Health (ET). Health Extension Implementation: Guide. Addis Ababa: Health Extension Education Center; 2007.

\section{THE AUTHOR}

Hailom Banteyerga (hailombante@ yahoo.com), specialist in health policy and systems analysis with a doctorate in education. Lead health researcher, MizHasab Research Center, Addis Ababa, Ethiopia.

Submitted: April 5, 2011

Approved for publication: June 28, 2011 Disclosures: None 